\title{
Deep Brain Stimulation may Reduce Tremor by Preferential Blockade of Slower Axons via Antidromic Activation
}

\author{
Míriam R. García, Mark Verwoerd, Barak A. Pearlmutter, Peter E. Wellstead, Richard H. Middleton
}

\begin{abstract}
Deep brain stimulation (DBS) has been used to ameliorate essential and Parkinsonian tremor, however the detailed mechanism by which tremor reduction is achieved remains unclear. We hypothesize that DBS works by reducing time delays in the feedback paths of the motor control loops. In particular, we suggest that antidromic activation of axonal pathways induced by stimulation will preferentially block axons with longer propagation times, reducing time delays in neuronal motor circuits in a stabilising manner. We demonstrate the plausibility of this hypothesis using two simple computational models which account for a variety of experimental results, and allow us to makes a number of testable predictions.
\end{abstract}

\section{INTRODUCTION}

About $60-70 \%$ of patients with idiopathic Parkinson's disease (PD) exhibit tremor, usually both resting and postural [1], [2]. It is believed that this pathological motor oscillation originates in the cortico-basal ganglia-thalamocortical or cerebello-thalamo-cortical motor circuits, but the precise details are unknown [3]. Nonetheless, both Parkinsonian and essential tremor have been successfully treated using a surgical technique called Deep Brain Stimulation (DBS), which consists of stimulating certain nuclei in the gangliathalamo-cortical pathway with a train of high frequency (HF) (typically slightly above $120 \mathrm{~Hz}$ ) electrical pulses [4]. However, the fundamental question of why this technique works is, as yet, unresolved.

From an engineering viewpoint, understanding the mechanism by which DBS works is important for the design of effective and efficient stimulation strategies. There are two two main approaches to this task. The first of these is based upon models of coupled oscillators simple enough that different control feedback mechanisms can be analyzed [5], [6]. The second main research strand involves developing sufficiently rich computational models of the basal ganglia (BG) [7], [8] to allow numerical studies of the closed loop problem. In addition to this, efforts have also been addressed to simulate the peripheral or/and the central nervous system by using transfer functions [9], [10], [11], [12], although usually without considering the effect of DBS on the central loops.

Early trials of DBS as a treatment option were carried out as an alternative to surgical lesion of certain parts of the brain, and has been referred to as 'informational lesion' procedure [4]. However, detailed analysis of electrical/neural interactions [13], [14] suggests that stimulators, rather than diminishing neural information flow, often produce an excitatory response. Based upon these observations, a number of mechanisms have been proposed, whose details depend on the relevant activated element (efferents, afferents and/or nearby fibers) [15], or on the observed effect in the BG network such as "jamming" of abnormal patterns [16] or desynchronization of the neural network [17].

In all of these theories, some basic features of DBS remain difficult to explain. For example, why is it that DBS is only effective at frequencies much higher than the tremor frequencies? And why is the therapeutic frequency range so wide? The location of the electrode and the time necessary to reduce the tremor are other issues that have not yet been convincingly rationalized. Despite the basic nature of these questions, they are often only considered in a peripheral manner. For example, to the best of the authors knowledge, there is only one work aiming to explain the need for HF stimulation using classical control theory [18].

In this article we hypothesize that DBS ameliorates tremor by shortening the communication delay in the cortico-basal ganglia-thalamocortical feedback loop. This hypothesis is inspired by the known influence of time delay on closed loop stability and based on several experimental observations. We will show also that this theory explains some of the basic features mentioned previously, such as the stimulation frequency and electrode location, and offers a new control systems perspective to the DBS problem.

The paper is organized as follows: in Section II we present the hypothesis, and illustrate its plausibility using two simple models: (i) a biomechanical model of the arm in Section III and (ii) a random neuronal network in Section IV. We conclude with proposals for further research.

\section{BLOCKADE HYPOTHESIS}

In this section, we explain how experimental evidence available in the literature justifies our assumptions and describe the blockade hypothesis and predictions.

\section{A. Stimulation causes antidromic activation of long axons}

One of the main reasons for the controversy about the working mechanism of DBS lies in the difficulty in determining which neuronal elements are activated by DBS. Some recent research has suggested that these results can be understood if DBS works by stimulating neuronal axons, but not neuronal somas [14], [13]. In fact the chronaxies ${ }^{1}$ of myelinated fibers vary in the range of 30-200 $\mu$ s, while cell bodies have chronaxies in the $1-10 \mathrm{~ms}$ band [19]. Since the usual pulse width in DBS is between 60-450 $\mu$ s (with more current required for the smallest widths), the larger

\footnotetext{
${ }^{1}$ Chronaxies are defined as the minimum interval of time necessary to electrically stimulate a fiber using twice the minimum current needed to elicit a threshold response.
} 
myelinated axons connecting different structures would tend to be activated rather than the cell bodies [13]. It should be noted that, if this is the case, then DBS will only excite the long axons (myelinated ones) connecting different parts of the brain and the behavior inside the stimulation structure can be neglected.

When we look at the form of activation, the results are more conclusive: electric pulses can travel in both directions from the stimulated axonal point. They can travel forward, that is toward the synaptic connection (orthodromically), or they can travel in the reverse direction toward the soma (antidromically). However, only low frequency stimulation can be decoded by the neurons. High frequency stimulation of the soma might only contribute by overriding pathological neuronal discharge imposing a more regular effect [13], [14]. For this reason we make the working hypothesis that DBS works primarily by antidromically activating long axons connecting different parts of the brain.

\section{B. Antidromic pulses may collide with spikes from the cortex}

We now discuss the likely results of antidromic stimulation of an axon. We focus on those parts of the brain where pathological oscillations are observed, (see Fig. 1). There are two main targets for tremor amelioration (referred to as Tremor Ameliorating Targets (TATs)): the Subthalamic Nucleus (STN) and the thalamus.

As can be seen in Fig. 1, several parts of the brain can be antidromically activated by stimulation of the TATs. However, recent work [20] has shown that stimulating axonal bundles connecting the cortex and STN in Parkinsonian rat models produced similar beneficial effects to simulation of the STN itself. This work plus the importance of the cortex in commanding both tremor related pathways (cortical-basal ganglia-thalamo-cortical and cerebello-thalamo-cortical) support the hypothesis that connections between the cortex and TATs are of key importance in understanding tremor amelioration by DBS.

The antidromic propagation in afferent fibers, or even in nearby fibers, seems to produce one or both of the following two effects: (i) stimulation of the projecting nuclei from TAT [21] and (ii) collision of antidromic spikes with spontaneous orthodromic spikes leading to the blockade of the orthodromic spikes [14], [13], [22]. There are several arguments to support the importance of the second mechanism: (a) usually, Parkinsonian brains are more energy stressed than normal brains[23]. Also, (b) a number of sources have reported the difficulty of exciting the neuronal soma by antidromic activation at HFs [24]. (c) Recent studies [22] of causality between the STN and the forearm muscles in PD found that the STN receive more afferences than it emitted efferences. Finally, (d) direct motor cortex stimulation is effective for suppressing akinesia, bradykinesia but not tremor [25]. Due to these observations, we assume that the beneficial effects of HF stimulation are consistent with blocking the axonal transmission rather than the activation of TAT downstream neural networks. However, the plausibility of this hypothesis

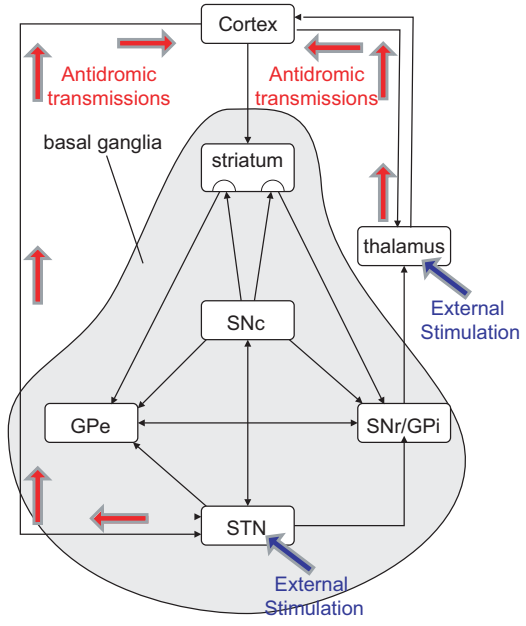

Fig. 1: DBS effects under the assumption of cortical projections to the possible Tremor Ameliorating Target (TAT): STN and thalamus.

and its connection with the high stimulation frequencies have not yet been studied.

\section{At HFs, DBS preferentially blockades slow axons}

It is well-known in neurology that long myelinated axons conduct traveling spikes at different velocities, and that this velocity is proportional to the axonal diameter. In fact, the following relations between propagation times and diameters are obtained from experimental data [26]:

$$
\tau_{i}=\frac{L}{v_{i}}=\frac{L}{\alpha D_{i}+\beta} \quad \text { with } \begin{aligned}
\alpha & =9.7 \times 2.15 \\
\beta & =9.7 \times 0.013
\end{aligned}
$$

where $\tau_{i}[\mathrm{~ms}], L[\mathrm{~mm}]$ and $v_{i}[\mathrm{~mm} / \mathrm{ms}]$ are the travelling times, length and velocities, respectively, and $i$ ranges from one to the number of fibers with different diameter. The parameters $\alpha$ and $\beta$ collect the linear relation found between velocity and diameter and the correction factor of the shrinkage of the diameter after fixing and embedding the tissue in paraffin.

Based on these observations, we posit that antidromic activation may partially blockade axonal pathways. We use the term partial since the blockade is less complete for axons with higher propagation velocities. This idea is illustrated for the case of the HF stimulation of a human brain in Fig. 2. The figure shows in schematic form the range of axonal thicknesses that exist within the axonal bundle linking the TAT and cortex. When subject to stimulation, antidromic stimulation completely blocks axons thinner than $0.4 \mu \mathrm{m}$. Axons thicker than $3 \mu \mathrm{m}$ allow almost all the beta oscillation from the cortex to pass and stimulate the TAT. In axons with diameter close to $1 \mu \mathrm{m}$, the influence of afferent stimulation is to perform the equivalent of a low pass filtering action upon the beta frequency.

\section{Partial blockade reduces the effective transmission delay}

To describe the effect of antidromic blockade, we build a distribution, or Probability Density Functions (PDF) for the 


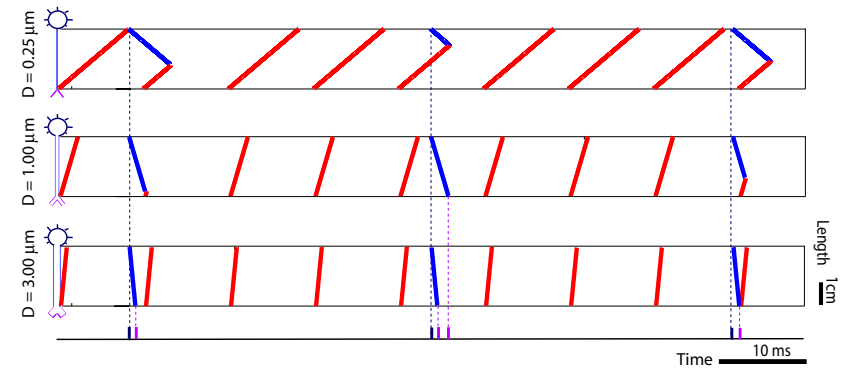

Fig. 2: DBS antidromic blockade is less effective for axons with greater diameter. Interaction is shown between orthodromic beta spikes and an antidromic DBS pulse train in axons of different diameters. Beta frequency somatic spikes $(29 \mathrm{~Hz})$ are shown in blue traveling orthodromically (downward), while antidromic spikes due to high frequency DBS $(103 \mathrm{~Hz})$ are shown in red. The differing diameters result in differing conduction velocities (top to bottom: $3.3 \mathrm{~mm} / \mathrm{ms}$, $13.4 \mathrm{~mm} / \mathrm{ms}$, and $40.1 \mathrm{~mm} / \mathrm{ms}$ ) which results in a higher proportion of spikes clearing the axon without interference in larger-diameter axons.

axonal diameters. From this PDF we derive a PDF for the transmission delay.

The axonal diameter PDF is obtained by rescaling observations of neural delay in other nerve bundles. Fig. 3a, was obtained from histograms of different diameters in the human midbody [26]. To obtain a smooth approximation to the PDF we use the method in [27] implemented in MATLAB (R2009a, The MathWorks) with normal kernel and restricting the density to positive values. Common measures of latency between TAT and the cortex are approximately $2 \mathrm{~ms}$ [28], [30], [21]. From this and the diameter PDF in 3a we obtain an estimate of the length of the pathway as $L=23.79 \mathrm{~mm}$. With these data, the resulting distribution of delays can be seen in Fig. 3a.

Let us denote by $\lambda$ the time between consecutive DBS pulses, the probability of blockade $(P)$ and transmission $(1-$ $P$ ) can be easily computed if noting that there is complete blockade if $2 \tau \geq \lambda$ :

$$
P=\left\{\begin{array}{ll}
1, & \text { if } 2 \tau \geq \lambda \\
\frac{2 \tau}{\lambda}, & \text { if } 2 \tau<\lambda
\end{array} .\right.
$$

The relationship between delay and blockade probability (2) is illustrated in Fig. 3b for several DBS frequencies.

Finally, by multiplying the delay PDF by the transmission probability at different stimulation frequencies, the PDFs in Fig. 3c are obtained. As can be seen, it is necessary to stimulate at frequencies greater than $110 \mathrm{~Hz}$ to achieve significant attenuation of transmissions with delay greater than $5 \mathrm{~ms}$.

\section{E. Predictions}

Two main predictions can be obtained from the partial blockade hypothesis: stimulation frequency should be high enough to clear the slower axons but not the faster ones,

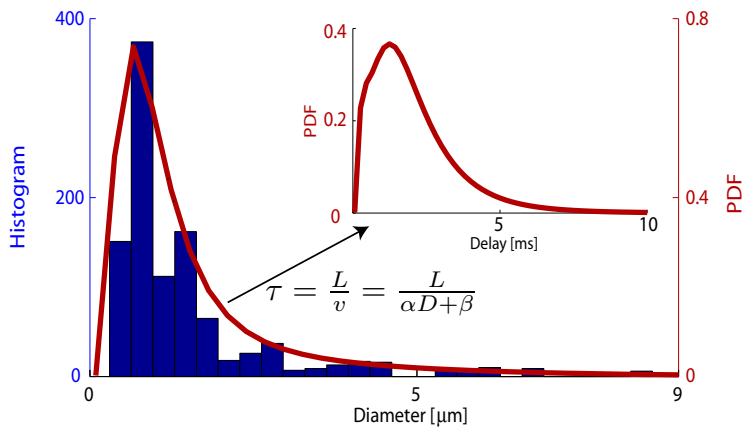

(a) Axonal diameter distribution in the human splenium [26]
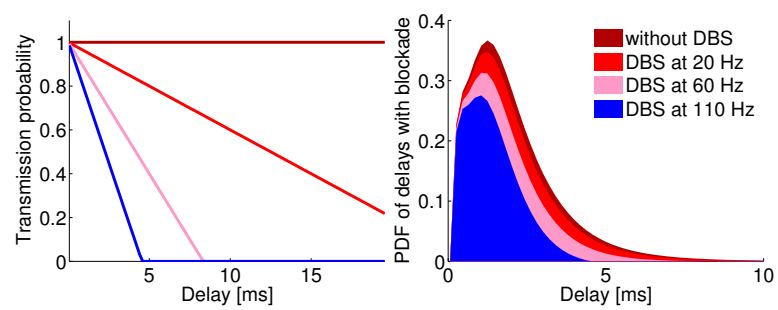

(b) Transmission probability

(c) PDF at different DBS frequencies

Fig. 3: Mean responses in the TAT to a cortical spike without and with DBS at different frequencies.

and the stimulation has to be applied to structures of brain motor loops receiving projections from the motor cortex. Axonal diameter distributions and pathway lengths can be measured using diffusion-weighted MRI [29]. Specifically, it can be used to test that: (a) bundles of axons traveling from the cortex to different TATs should have similar delay distributions, i.e., similar relationship between the length and the diameter and even with the degree of myelin. (b) Where there are substantial differences in the minimum effective DBS frequency, there should also be differences in the delay distribution of the stimulated pathway. If this observation is confirmed then, pre-clinical studies can be done to estimate the optimal stimulation frequency before inserting the DBS probe.

In the literature we can find experimental evidence supporting these predictions. For instance, the three structures that receive projections from the cortex (see Fig. 1) are the STN, thalamus and striatum. A large number of experiments have shown that the STN and the thalamus receive information from the same cortical layers and both may ameliorate tremor, but the picture is much more complicated for the striatum. Moreover, similar delays have been measured from the rat STN [21], human STN [28] and mice thalamus [30] to the cortex.

Moreover, although the globus pallidus internus (GPi) is usually targeted for the treatment of Parkinsonian symptoms other than tremor, in some cases it may also ameliorate tremor [31]. It should be note that this agrees with the hypothesis since cortical fibers pass close to the GPi and can thus be indirectly activated by DBS [21].

Finally, it has also been observed that it is possible to suppress Parkinsonian tremor by stimulating the spinal cord 
at $300 \mathrm{~Hz}$ [32]. This experiment has two noteworthy features: (i) the frequency of stimulation is more than double that used in other target areas and (ii) the electrode is located in the sensory fibers of the spinal cord and not in a nucleus of the BG. Both of these features can be again interpreted in the framework of the hypothesis. First, the majority of the spinal cord sensory fibers go to the brainstem, and it has been demonstrated using spike collisions that the sensory pathway connecting the brainstem with the cortex shares a common segment with the pathway connecting the thalamus with the cortex [24]. Second, since the stimulation frequency is between two and three times higher than that usual in conventional DBS target sites, the shared pathway should be between two and three times shorter than the thalamus-cortex pathway. Both of these predictions are testable.

\section{BIOMECHANICAL MODEL}

In this section, we use a basic control model to argue that reducing the effective delay of the feedback loop, the system can be stabilized and that this change of behavior agrees with amplitude and frequency changes in tremor when DBS is turned on.

As background, we first review a known result from control theory: that a communication delay in the feedback path of a control system can have a destabilizing effect [33]. Fig. 4 is a simple biomechanical model of limb angle under the action of torque $T$ induced by a motor control circuit. We assume that the motor control circuit uses a generic control structure (PID, or proportional, integral plus derivative [34]) control to maintain the limb at rest in a horizontal position against gravity. Fig. 5 shows the behavior of the closed-loop system as a function of the delay parameter.

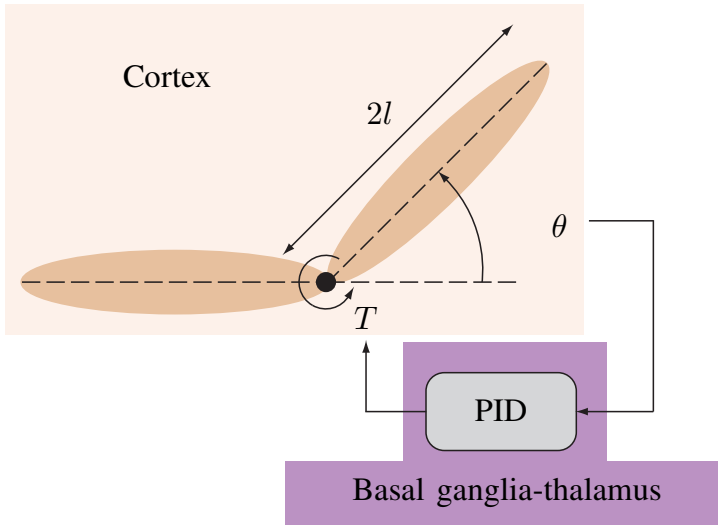

Fig. 4: A simple biomechanical model of a lower limb and its mirror in the brain loops.

For the biomechanical model depicted in Fig. 4, the equations of motion are

$$
\ddot{\theta}(t)=-\frac{g}{l} \cos \theta(t)+\frac{1}{m l^{2}} T(t) .
$$

where $\theta(t)$ denotes the joint angle as a function of time, $g=$ $10 \mathrm{~ms}^{-2}$ is the local acceleration due to gravity, $m=2 \mathrm{~kg}$ is the mass of the limb, $l=25 \mathrm{~cm}$ is the distance from the joint to the center of mass and $T(t)$ is the applied torque. We assume that the torque exerted is a control force, of the form

$$
\begin{gathered}
T(t)=k_{p} \sin \theta(t-\tau)+k_{d} \operatorname{atan} \alpha_{d} \dot{\theta}(t-\tau) \\
+k_{i} \text { atan } \alpha_{i} \int_{-\tau}^{t-\tau} \theta\left(t^{\prime}\right) \mathrm{d} t^{\prime}
\end{gathered}
$$

where $k_{p}=100, k_{d}=30, k_{i}=40$ are the proportional, derivative and integral controller constants, $\tau>0$ is a fixed delay associated with motor circuit control processing. The function atan models saturation and $\alpha_{d}$ and $\alpha_{i}$ are scaling factors.

We observe that in the absence of delay, the limb quickly returns to rest following its release from a horizontal position (panel A). Qualitatively similar behavior is observed when small but nonzero time delays are used. However, when the delay is increased beyond a certain critical value $(6.2 \mathrm{~ms}$ for the parameter values adopted here) the system no longer returns to rest, but rather enters a stable oscillation around the equilibrium point (panels $\mathbf{C}$ and $\mathbf{D}$ ). The amplitude and the frequency of this oscillation depend upon the value of the delay parameter in a predictable manner, with a larger delay corresponding to a lower frequency and a higher amplitude respectively (panel B). This behavior is characteristic of a well-known phenomenon in the theory of dynamical system known as a (supercritical) Hopf bifurcation [35], the same bifurcation observed in the models simulating the competition between feedback loops in the BG [36]. We note that the stable regime is finite: further increasing the delay parameter will lead to a second bifurcation that renders the oscillations unstable. This phenomenon is also extremely robust to the particular details of the controller.

The main predictions obtained from the model are (i) the reduction of tremor amplitude and (ii) the increase of the tremor frequency. The first prediction coincides with the experimental observation when DBS is applied in the therapeutic range, the tremor amplitude decreases in seconds and always within one minute [37]. The impact of DBS upon tremor frequency has only been studied in detail by a few groups, with the data supporting our prediction. For example, the ventral intermediate nucleus of the thalamus has been stimulated in order to suppress essential tremor associated with the olivary nucleus and the cerebellum [38]. In that study, DBS increased the tremor frequency for different patients from $4 \mathrm{~Hz}$ to $5 \mathrm{~Hz}$ whenever the load was not higher. Similar results have been obtained when stimulating the STN to alleviate Parkinsonian tremor [2], [37] in postural and resting tasks. In both types of tremor, the amplitude decreased with DBS or/and medication. However, the frequency measured (peak frequency of the extensor digitorum electromyography signal between $1-9 \mathrm{~Hz}$ ) increased with STN DBS but not with medication.

It should be stressed that although this result agrees with experimental observations, it is only illustrative of tremor amelioration. In reality, the basic control law for arm motion uses feedback from the peripheral nervous system and DBS 

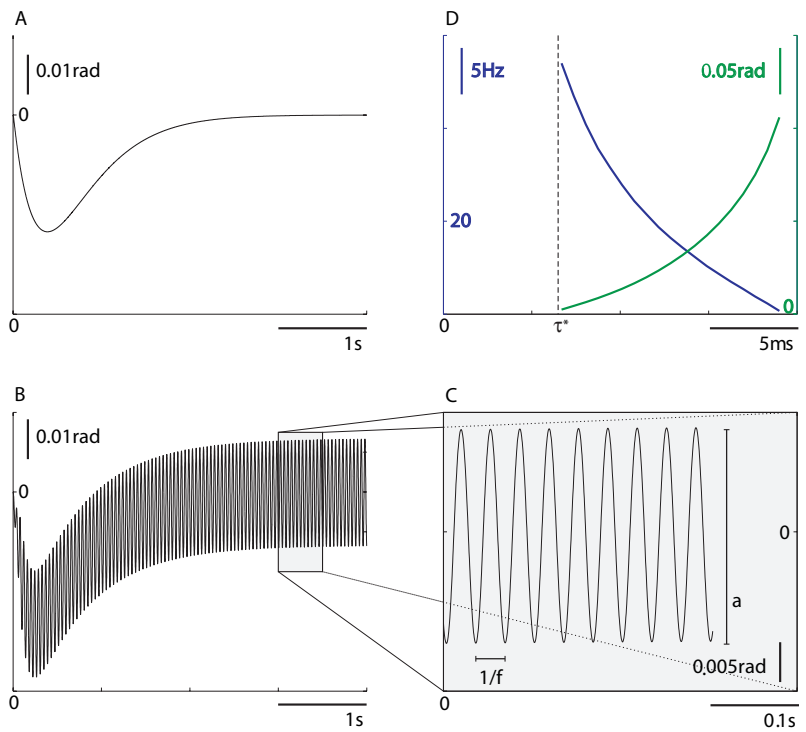

Fig. 5: (A) Closed-loop control is used to regulate joint angle at the horizontal position $\theta(t)=0$. (B) At a larger delay around the control loop, however, the joint angle changes from stable control to oscillatory. The existence of such a transition $\tau^{*}$ is extremely robust, being determined by the size of the time delay in the loop-it is largely independent of the details of the system or controller. (C) Zoom of the oscillatory behaviour. (D) As the delay control loop increases, for values larger than the critical value $\tau^{*}$, the frequency of the oscillation decreases and the amplitude increases

acts in the central nervous system. However, it is known that the arm movements are mirrored in specific areas of the motor cortex. A better model might consider the more complex case where the cortex simulates the arm movements and the BG and thalamus act as an internal controller, adapting the movement and selecting those set points to be sent to the peripheral nervous system [39].

\section{A RANDOM NEURONAL NETWORK WITH DIFFERENT DELAYS}

We now re-examine the antidromic blockade hypothesis in a random neuronal network with several delayed connections. The aim of this is to show that the results are not dependent on the specific details and organization of the BG circuit and apply to other alternate models. For this purpose, we simulate excitatory and inhibitory neurons modeled by the following equations:

$$
\begin{gathered}
\dot{v}=0.04 v^{2}+5 v+140-u+I, \quad \dot{u}=a(b v-u) \\
\text { if } v \geq+30 \mathrm{mV}, \text { then }\left\{\begin{array}{l}
v \leftarrow c \\
u \leftarrow u+d
\end{array}\right.
\end{gathered}
$$

Due to lack of space, the complete model is not given here. In summary, we use the model of [40] with several changes to simulate the Parkinsonian state: (1) we use 3 excitatory

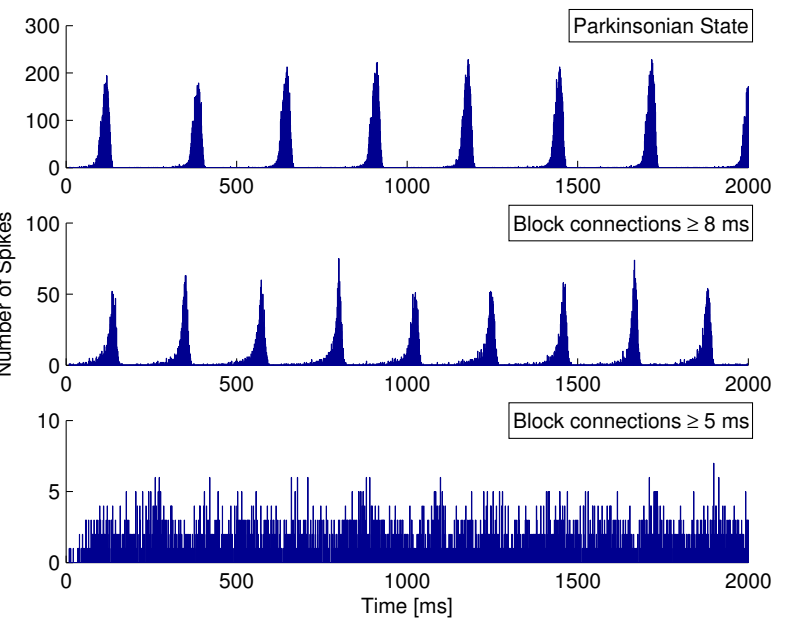

Fig. 6: Neural Network in Parkinsonian State under blockade of slow connections.

synapses to represent synchronized pathological behavior; (2) we ignore neuronal plasticity due to the lack of dopamine in the PD situation, and (3) delays are kept between 1 and $10 \mathrm{~ms}$ to consider connections among distant neurons such as those connecting TATs with the cortex.

Under these circumstances, as can be seen in Fig. 6, blockade of connections with delays greater than $8 \mathrm{~ms}$ changes the frequency and amplitude of the histograms. More interestingly, when delays greater than $5 \mathrm{~ms}$ are blocked we observe not only a change in frequency but also a desynchronization of the network. This agrees with current hypotheses that DBS works by desynchronizing the pathological synchronized oscillations in the BG.

\section{CONCLUSIONS AND FUTURE WORK}

\section{A. Conclusions}

Based on experimental observations, we propose a new hypothesis that might explain how DBS ameliorates tremor. The idea is based on a known result from control theory: that in many feedback loops, the reduction of communication delay in a feedback path improves stability. Two simple models have been used to illustrate the idea: a biochemical model of the arm movement and a random neuronal network. Both models show a change of behavior under DBS that agrees with several experimental observations.

In addition, we think that this hypothesis could motive a new way of looking at this open problem where theoretical control analysis would be fundamental.

\section{B. Future Works}

Both models presented here illustrate the main idea of the hypothesis and show some qualitative testable predictions. However a more realistic model of the cortico-basal gangliathalamocortical loop circuit might give us specific quantitative predictions that can be examined experimentally. Such a model would need to differentiate between the different 
nuclei and connections in the BG-thalamocortical circuit and might include different types of neuronal behaviors such as bursting.

\section{REFERENCES}

[1] G. Grimaldi and M. Manto, "Tremor: From pathogenesis to treatment," Synthesis Lectures on Biomed. Eng., vol. 3, no. 1, pp. 1-212, 2008.

[2] C. Blahak, J. C. Wöhrle, H.-H. Capelle, H. Bäzner, E. Grips, R. Weigel, M. G. Hennerici, and J. K. Krauss, "Tremor reduction by subthalamic nucleus stimulation and medication in advanced Parkinson's disease," J. Neurol., vol. 254, no. 2, pp. 169-78, 2007.

[3] G. Deuschl, J. Raethjen, R. Baron, M. Lindemann, H. Wilms, and P. Krack, "The pathophysiology of Parkinsonian tremor: A review," $J$. Neurol., vol. 247, no. 17, pp. 33-48, 2000.

[4] A. L. Benabid, "Deep brain stimulation for Parkinson's disease," Curr Opin Neurobiol, vol. 13, no. 6, pp. 696-706, 2003.

[5] O. V. Popovych, C. Hauptmann, and P. A. Tass, "Effective desynchronization by nonlinear delayed feedback," Phys. Rev. Lett., vol. 94, no. 16, p. 164102, 2005.

[6] P. Danzl and J. Moehlis, "Event-based feedback control of nonlinear oscillators using phase response curves," in Decision and Control, 2007 46th IEEE Conference on. IEEE, 2008, pp. 5806-11.

[7] S. Santaniello, G. Fiengo, L. Glielmo, and W. M. Grill, "Basal ganglia modeling in healthy and Parkinson's disease state. i. isolated neurons activity," in American Control Conference, 2007. ACC'07. IEEE, 2007, pp. 4089-94.

[8] S. Santaniello, G. Fiengo, and L. Glielmo, "Basal ganglia modeling in healthy and Parkinson's disease state. ii. network-based multi-units simulation," in American Control Conference, 2007. ACC'07. IEEE, 2007, pp. 4095-100.

[9] R. E. Suri, C. Albani, and A. H. Glattfelder, "A dynamic model of motor basal ganglia functions," Biol. Cybern, vol. 76, no. 6, pp. 451-8, 1997.

[10] M. Haeri, Y. Sarbaz, and S. Gharibzadeh, "Modeling the Parkinson's tremor and its treatments," Journal of theoretical biology, vol. 236, no. 3, pp. 311-22, 2005

[11] M. MashhadiMalek, F. Towhidkhah, S. Gharibzadeh, V. Daeichin, A. P. Ali, et al., "Are rigidity and tremor two sides of the same coin in Parkinson's disease?" Computers in Biology and Medicine, vol. 38, no. 11-12, pp. 1133-9, 2008.

[12] D. Zhang, P. Poignet, A. P. L. Bo, and W. T. Ang, "Exploring peripheral mechanism of tremor on neuromusculoskeletal model: A general simulation study," IEEE Trans. Biomed. Eng., vol. 56, no. 10, pp. 2359-69, 2009.

[13] C. Hammond, R. Ammari, B. Bioulac, and L. Garcia, "Latest view on the mechanism of action of deep brain stimulation," Mov Disord, vol. 23 , no. 15 , pp. $2111-21,2008$.

[14] M. D. Johnson, S. Miocinovic, C. C. McIntyre, and J. L. Vitek, "Mechanisms and targets of deep brain stimulation in movement disorders," Neurotherapeutics, vol. 5, no. 2, pp. 294-308, 2008.

[15] T. Heida, E. Marani, and K. G. Usunoff, The subthalamic Nucleus, Part II: Modelling and Simulation of Activity, ser. Advances in Anatomy, Embryology, and Cell Biology. Berlin, Heidelberg: Springer-Verlag, 2008, vol. 199.

[16] C. C. McIntyre, M. Savasta, L. Kerkerian-Le Goff, and J. L. Vitek, "Uncovering the mechanism(s) of action of deep brain stimulation: Activation, inhibition, or both," Clin. Neurophysiol., vol. 115 , no. 6 , pp. 1239-48, 2004.

[17] P. Tass, "Effective desynchronization with a resetting pulse train followed by a single pulse," EPL (Europhysics Letters), vol. 55, p. $171,2001$.

[18] A. M. de Paor and M. A. Lowery, "Analysis of the mechanism of action of deep brain stimulation using the concepts of dither injection and the equivalent nonlinearity," IEEE Trans. Biomed. Eng., vol. 56, no. 11, Part 2, pp. 2717-20, 2009.

[19] J. B. Ranck, Jr., "Which elements are excited in electrical stimulation of mammalian central nervous system: A review," Brain Res., vol. 98, no. 3, pp. 417-40, 1975.

[20] V. Gradinaru, M. Mogri, K. R. Thompson, J. M. Henderson, and K. Deisseroth, "Optical deconstruction of Parkinsonian neural circuitry," Science, vol. 324, no. 5925, pp. 354-9, 2009.
[21] S. Li, G. W. Arbuthnott, M. J. Jutras, J. A. Goldberg, and D. Jaeger, "Resonant antidromic cortical circuit activation as a consequence of high-frequency subthalamic deep-brain stimulation," J. Neurol., vol. 98, no. 6, pp. 3525-37, 2007.

[22] E. Florin, J. Gross, C. Reck, M. Maarouf, A. Schnitzler, V. Sturm G. R. Fink, and L. Timmermann, "Causality between local field potentials of the subthalamic nucleus and electromyograms of forearm muscles in Parkinson's disease," Eur J Neurosci, vol. 31, no. 3, pp. 491-8, 2010.

[23] P. Wellstead and M. Cloutier, "An energy systems approach to Parkinson's disease," WIREs Systems Biology and Medicine, vol. 3, no. 1, pp. 1-6, 2011.

[24] T. Chomiak and B. Hu, "Axonal and somatic filtering of antidromically evoked cortical excitation by simulated deep brain stimulation in rat brain," J Physiol, vol. 579, no. 2, pp. 403-12, 2007.

[25] X. Drouot, S. Oshino, B. Jarraya, L. Besret, H. Kishima, P. Remy, J. Dauguet, J. P. Lefaucheur, F. Dollé, F. Condé, M. Bottlaender, M. Peschanski, Y. Kéravel, P. Hantraye, and S. Palfi, "Functional recovery in a primate model of Parkinson's disease following motor cortex stimulation," Neuron, vol. 44, no. 5, pp. 769-78, 2004.

[26] F. Aboitiz, A. B. Scheibel, R. S. Fisher, and E. Zaidel, "Fiber composition of the human corpus callosum," Brain Res., vol. 598, no. 1-2, pp. 143-53, 1992

[27] A. W. Bowman and A. Azzalini, Applied smoothing techniques for data analysis: the kernel approach with S-Plus illustrations. Oxford University Press, USA, 1997.

[28] K. B. Baker, E. B. Montgomery, Jr., A. R. Rezai, R. Burgess, and H. O Lüders, "Subthalamic nucleus deep brain stimulus evoked potentials: Physiological and therapeutic implications," Mov Disord, vol. 17, no. 5, pp. 969-83, 2002

[29] D. Barazany, P. J. Basser, and Y. Assaf, "In vivo measurement of axon diameter distribution in the corpus callosum of rat brain," Brain, vol. 132, no. 5, pp. 1210-20, 2009.

[30] M. Salami, C. Itami, T. Tsumoto, and F. Kimura, "Change of conduction velocity by regional myelination yields constant latency irrespective of distance between thalamus and cortex," Proc. Natl. Acad. Sci. U. S. A., vol. 100, no. 10, pp. 6174-9, 2003.

[31] J. A. Obeso et al., "Deep-brain stimulation of the subthalamic nucleus or the pars interna of the globus pallidus in Parkinson's disease," $N$ Engl J Med, vol. 345, no. 13, pp. 956-63, 2001.

[32] R. Fuentes, P. Petersson, W. B. Siesser, M. G. Caron, and M. A. L. Nicolelis, "Spinal cord stimulation restores locomotion in animal models of Parkinson's disease," Science, vol. 323, no. 5921, pp. 1578 82, 2009.

[33] K. Gopalsamy, Stability and oscillations in delay differential equations of population dynamics. Springer, 1992.

[34] G. C. Goodwin, S. F. Graebe, and M. E. Salgado, Control System Design. Prentice Hall, 2001.

[35] E. M. Izhikevich, Dynamical Systems in Neuroscience. MIT Press, 2007.

[36] A. Leblois, T. Boraud, W. Meissner, H. Bergman, and D. Hansel, "Competition between feedback loops underlies normal and pathological dynamics in the basal ganglia," $J$ Neurosci, vol. 26, no. 13, pp 3567-83, 2006.

[37] C. Blahak, H. Bäzner, H.-H. Capelle, J. C. Wöehrle, R. Weigel, M. G. Hennerici, and J. K. Krauss, "Rapid response of Parkinsonian tremor to STN-DBS changes: Direct modulation of oscillatory basal ganglia activity?" Mov Disord, vol. 24, no. 8, pp. 1221-5, June 152009.

[38] D. E. Vaillancourt, M. M. Sturman, L. V. Metman, R. A. E. Bakay, and D. M. Corcos, "Deep brain stimulation of the VIM thalamic nucleus modifies several features of essential tremor," Neurology, vol. 61, no. 7 , pp. 919-25, 2003.

[39] E. R. Kandel, J. H. Schwartz, T. M. Jessell, S. Mack, and J. Dodd Principles of neural science, 3rd ed. Elsevier New York, 1991.

[40] E. M. Izhikevich, "Solving the distal reward problem through linkage of STDP and dopamine signaling," Cereb. Cortex, vol. 17, no. 10, pp. 2443-52, 2007. 